\title{
Virtual reality distraction for patients to relieve pain and discomfort during colonoscopy
}

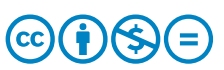

\author{
Authors \\ Institutions \\ 1 Department of Gastroenterology and Hepatology, \\ Radboud University Medical Centre, Nijmegen, the \\ Netherlands \\ 2 Department of Radiology and Nuclear Medicine, \\ Radboud University Medical Centre, Nijmegen, the \\ Netherlands \\ 3 Department of Biomedical Signals and Systems, \\ University of Twente, Enschede, the Netherlands
}

Govert Veldhuijzen*, ${ }^{*}$, Nienke J.M. Klaassen ${ }^{*}, 2$, Richard J.A. Van Wezel ${ }^{3}$, Joost P.H. Drenth ${ }^{1}$, Aura A. Van Esch ${ }^{1}$

submitted 21.11.2019

accepted after revision 16.4 .2020

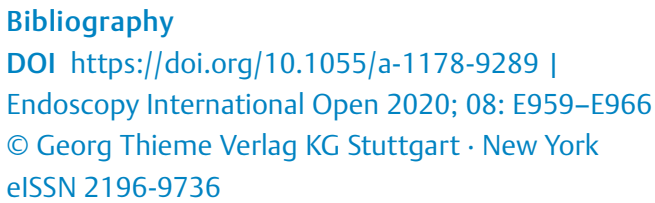

Corresponding author

Govert Veldhuijzen, Department of Gastroenterology and Hepatology, Radboud University Medical Centre, Nijmegen, the Netherlands

Fax: +31-243635129

Govert.Veldhuijzen@radboudumc.nl

\begin{abstract}
Background and study aims Colonoscopy is an invasive procedure that may cause patients pain and discomfort. Routine use of sedation, while effective, is expensive and requires logistical planning. Virtual reality (VR) offers immersive, three-dimensional experiences that distract the attention and might comfort patients. We performed a pilot study to investigate the feasibility of VR distraction during colonoscopy.
\end{abstract}

Patients and methods Adults referred for colonoscopy were considered for inclusion and divided over two groups: with and without VR glasses. The main outcome was patient acceptance of wearing VR glasses during colonoscopy without compromising the technical success of the procedure. Secondary outcomes were patient comfort, pain, and anxiety before, during and after the procedure, using validated patient questionnaires. Patient comments were collected through a qualitative interview.

Results We included 19 patients, 10 of whom were offered VR glasses. All patients accepted VR glasses without prolonging procedural time. No disadvantages of the VR glasses were reported in terms of communication or change of position of the patient. We found that patient comfort, pain, anxiety, and satisfaction in relation to the procedure were similar in both groups. Patients described a pleasant distracting effect using VR glasses.

Conclusion VR glasses during colonoscopy are accepted by patients and do not compromise endoscopic technical success. Patients reported that the VR experience was pleasant and distracting.

\section{Introduction}

Use of colonoscopy as a diagnostic and therapeutic tool is likely to rise. This is mainly a result of implementation and expansion of colorectal cancer screening projects, targeting ever younger patients [1]. Endoscopic procedures are associated with embarrassment, pain, and discomfort [2]. This proves an important barrier to undergoing colonoscopy and may subsequently

\footnotetext{
* Both authors contributed equally to this manuscript.
}

make patients less willing to be subjected to repeat surveillance colonoscopies [3, 4].

Indeed, a relevant proportion of patients (18\% to $29 \%$ ) experience anxiety due to concerns related to preparation for, execution of, and anticipation of the result of colonoscopy [5]. Sedation to relieve anxiety is the method of choice used to mitigate the discomfort patients experience during colonoscopy [6]. However, drug-induced sedation comes with adverse effects related to suppression of pulmonary and circulatory function $[7,8]$. There is a higher post-procedural risk of pneumonia in elderly patients [9]. Deep sedation even puts patients at in- 
creased risk for procedure-related complication of perforation [10]. Also, monitoring patients during and after sedation is both logistically demanding and costly [11].

Therefore, several studies have examined non-pharmacological interventions to reduce anxiety and pain during endoscopy [12-18]. These studies used a mix of visual $[13,15,18$, $19]$ or auditory stimuli $[12,14,18]$ and found that while true efficacy is not fully established, combined visual and auditory distraction is better at reducing discomfort than auditory distraction alone [18].

Virtual reality (VR) integrates computer-generated visual and auditory signals to recreate an illusionary perception of the actual physical world $[20,21]$. The distraction that comes with immersive VR induces an analgesic effect and has been used as an adjunct to control pain and anxiety during operative procedures [22,23]. VR technology has become more affordable and portable, adding to its immersive qualities [24]. VR reduces pain during burn wound debridement [25-27], and discomfort during dental procedures [28].

A questionnaire study found that up to $25 \%$ patients are willing to undergo colonoscopy with VR glasses instead of sedation. Key patient motive is the reduction in sedative use, which allows patients to drive their cars home themselves afterwards [29].

But still unknown is patient acceptance (e.g. feasibility) of performing colonoscopy on patients actually wearing VR glasses. Wearing VR glasses could potentially be disadvantageous to the colonoscopy procedure, as it could obstruct communication with patients. Conversely, the procedure itself might compromise the VR effect, as positional changes of the patient are sometimes necessary. Therefore, we set out in this pilot study to investigate use of VR distraction during colonoscopy. The primary aim was to assess patient acceptance of wearing VR glasses while undergoing colonoscopy. We were also interested in whether VR reduces discomfort, pain, and anxiety and increases satisfaction in patients compared to the standard practice.

\section{Patients and methods}

This experiment was designed as a pilot study to evaluate patient acceptance and practical feasibility. A control group was designed to allow evaluation of procedural and patient-related outcomes. The sample size was set at 12 subjects per group. This computation was based on a rule of thumb for pilot studies [30]. Ethical permission from the Radboudumc Ethics Committee was obtained prior to commencement of the study (number 2016-2750). The trial was registered with the Dutch trial Registry (NTR6175).

\section{Patients}

We screened patients who were already scheduled for outpatient colonoscopy. Inclusion criteria of the study were adult age and any elective indication of colonoscopy. Exclusion criteria of the study were visual and/or auditory impairments, dementia, limited Dutch language skills, and a diagnosis of balance disorders or epilepsy.
After evaluation of the above criteria, informed consent was obtained from all participants and they were allocated to the VR (intervention) or non-VR (control) group. Allocation was based on the day the colonoscopy was planned. Participants were informed about the group to which they were allocated on the day of the procedure.

\section{Intervention}

The hardware we used to generate VR distraction was the Samsung Gear VR (Consumer Edition-SM-R322, combined with Galaxy S7). This is an inexpensive (\$172) off-the-shelf wide fieldof-view, three-dimensional VR headset that projects video and rendered graphics into two independent lenses. The current model is the size of a small pair of ski goggles, with a combined weight of $470 \mathrm{~g}$, and is positioned on the head with elastic straps. The video content that was visualized on the VR hardware consisted of several short clips (with a total length 19 minutes, 59 seconds) of moving 360-degree cameras featuring tropical islands and forests in the Caribbean (supported by VR firm Visyon, Eindhoven, The Netherlands). The VR content had not audio, to allow optimal communication with the patient. The authors considered the chosen VR content to be of a relaxing and not overly thrilling character, generating an adequate level of distraction for all participants.

\section{Study design}

At T1, all participants filled out a baseline form on a tablet with information about demography, prior experience undergoing colonoscopy, prior VR experience, and a validated general health questionnaire (RAND-36) [31]. On T2, the day of colonoscopy, about 15 minutes before the procedure, all patients received a second form that included validated questionnaires on anxiety (STAI) and pain (NRS) [32,33]. Patients in the intervention group also tested the VR glasses before colonoscopy. During colonoscopy, T3, one researcher (NK) observed the patient's well-being and positioning together with several procedural aspects e.g., time to cecul intubation and time of total procedure. All patients received conscious sedation with midazolam and/or alfentanyl according to the standard of care, with the dose increased at physician discretion. After colonoscopy, patients completed a set of questionnaires at T4, including questions about anxiety (STAI), pain (NRS), net promoter score, and willingness-to-return questions. A short qualitative interview was held with the patients in the intervention group to explore their experiences with VR glasses.

\section{Measures}

Primary outcome

The main outcome was patient acceptance of wearing VR glasses during the procedure. That included adequate positioning of the VR glasses during the entire procedure, even during patient repositioning. In addition, we recorded cecal intubation rate, cecum and total procedure time as well as administered sedatives and analgesics. 


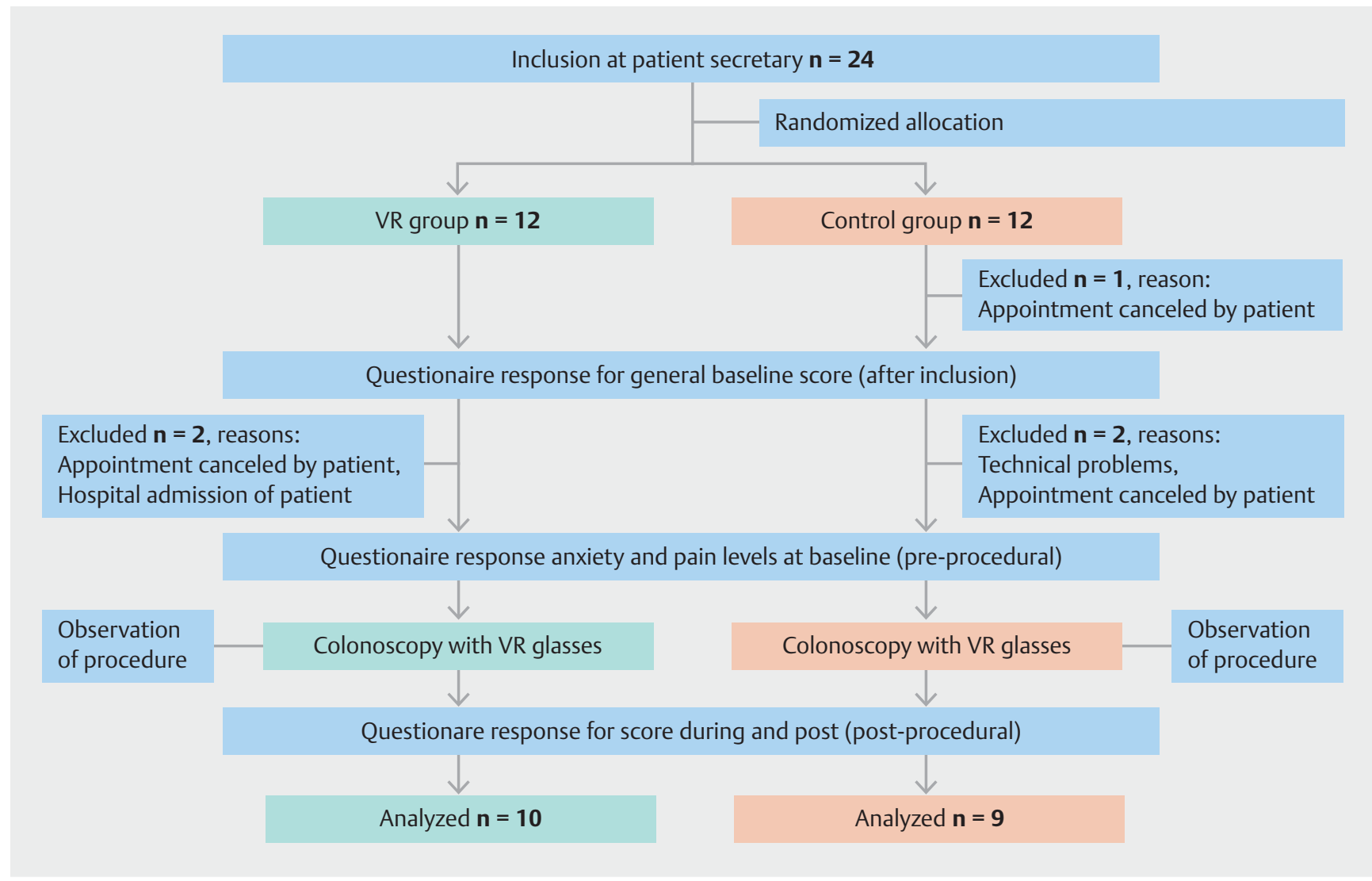

- Fig. 1 Study flowchart.

\section{Secondary outcomes}

Patient comfort

Patient comfort was measured using a five-point Gloucester Comfort scale: 1, comfortable and 5, severe discomfort [34].

\section{Patient pain}

An 11-point numeric rating scale (NRS) was used to measure pain of the patient before and during the procedure: 0 , no pain and 10, highest imaginable pain [32].

\section{Patient anxiety}

The State Trait Anxiety Inventory (STAI) was used to measure patient anxiety before and after the procedure. The 20-item STAl is widely used with scores ranging from 20 (absence of anxiety) to 80 (high anxiety) [33].

\section{Patient satisfaction}

The general health of the participants was measured using the RAND 36 questionnaire [31]. Net Promoter Score (NPS) [35] and an 11-point scale of willingness to return: 0 , no willing at all and 10 , definitely willing, were used to measure participant satisfaction with the procedure.

\section{Statistical analysis}

Statistical analysis was performed using SPSS version 22 (International Business Machines Corporation, Armonk, New York, United States). Mann Whitney U-tests were used to test whether the median scores for, i.e., age, pain, dose of medication, duration of the procedure, anxiety, satisfaction, NPS, and willingness to return, of the VR (intervention) and non-VR (control) group were comparable to each other. Fisher's Exact tests were used to test categorical data. $P \leq 0.05$ was considered statistically significant.

\section{Results}

In total 24 patients entered the trial ( $\triangleright$ Fig. 1 ). Patients were recruited at the endoscopy outpatient clinic. There were 55 eligible patients scheduled for colonoscopy within a 4-week timeframe and we invited 38 consecutive patients, 24 of whom accepted our invitation. Informed consent was obtained from all patients. After allocation, two patients in the VR (intervention) group and three patients in the non-VR (control) group were excluded (three patients cancelled the scheduled appointment, one was admitted to the hospital, and in one patient there was a technical problem with the endoscopy equipment). As a result, 19 patients were included in the final analysis, 10 in the VR group and nine in the non-VR group. All patients in the intervention group used the VR glasses during the whole procedure 


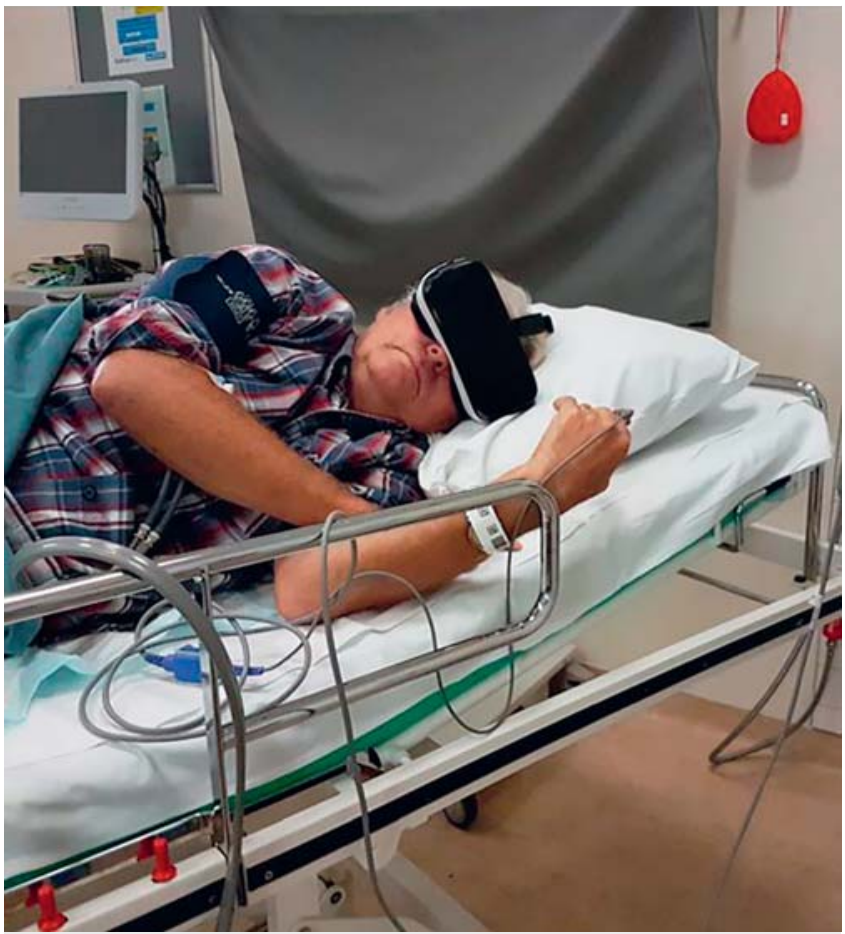

- Fig. 2 Samsung Gear VR shown on a patient during colonoscopy (with permission).

( $\triangleright$ Fig.2). No adverse events associated with VR distraction in combination with medication were observed. One endoscopist performed all the procedures (FV) except one in the VR group $(\mathrm{BvH})$. FV had $>5$ years of experience, $\mathrm{BvH}>3$ years.

\section{Baseline characteristics}

No significant differences were observed in baseline characteristics of the two groups, i.e., gender (55.6\% women in the control group, versus $60 \%$ women in the intervention group), age (median, 64, versus 65 years), level of education, RAND-36, previous colonoscopy, and prior experience with VR ( $\triangleright$ Table 1 ).

\section{Procedure characteristics}

There were no differences in procedural characteristics. The time to reach the cecum (median 10.48 minutes in the control group, versus 6.83 minutes in the intervention group), time to complete procedure (median 21.20 minutes in the control group, versus 22.60 minutes in the intervention group), and completed colonoscopies ( $100 \%$ in the control group, versus $90 \%$ in the intervention group) were comparable in the two groups ( $\triangleright$ Table 2 ).

Similarly, both groups were comparable in terms of initial intravenous bolus of sedatives and analgesics, i.e., dose of midazolam (median, $2.5 \mathrm{mg}$ in both groups), dose of alfentanyl (median, $0.25 \mathrm{mg}$ in both groups).

\section{Patient pain, comfort and anxiety}

Results of pain scores, patient comfort, and anxiety scores are summarized in $>$ Table 3 . Median pain score before (0 in both groups) and during ( 3 in both groups) the procedure was sim- ilar in both groups. The Gloucester Comfort scale did not reveal significant differences in patient comfort between the two groups (4 patients [44\%] in the control group were rated comfortable, versus 4 patients [ $40 \%$ ] in the intervention group). No significant difference was observed in median anxiety score prior to the procedure (49 in the control group, versus 48.5 in the intervention group). Median baseline anxiety score (trait) was similar in the intervention group and the control group (29 in the control group, versus 35 in the intervention group). Median anxiety score increased after the procedure (50 and 50).

\section{Patient satisfaction}

No differences were observed between the two groups in patient satisfaction. All patients scored high satisfaction rates in the scales that were used (median score was of 9 out of 10 in both groups). Results on patient satisfaction are summarized in Table 4.

\section{Qualitative comments}

The majority of patients (9/10) rated use of VR glasses as positive. Four patients indicated that they preferred to select the VR content themselves. Two patients complained about the quality of the movie and one patient indicated that the resolution of the VR movie was too low. The physician who performed the colonoscopy was able to communicate with all patients in the intervention group and did not experience any limitations with use of VR.

\section{Discussion}

Our pilot study shows that it feasible to use VR distraction during colonoscopy as we observed complete patient acceptance of the device during all procedures. Procedural time was not longer as a result of our intervention.

Comfort, pain, anxiety and patient satisfaction were not affected by VR, but patients reported a positive distracting effect of the VR glasses.

This pilot study indicates that there are no obstacles to investigating VR glasses further in a larger sample of patients. Important to the design of subsequent trials from the endoscopist perspective is that use of VR glasses did not interfere with the completion colonoscopy.

Various studies have found that visual and/or auditory distraction during endoscopic procedures reduces pain and improves satisfaction as a result $[13,15,18]$. In this pilot we were not able to identify these advantages for VR. This is similar to outcomes in two trials of VR in burn wound victims in which the authors suggested resolving this issue by developing a better-customized VR system instead of off-the-shelf VR sets [36, 37].

Indeed, patients reported that the effect of the VR distraction was less immersive probably because of the content shown. Other studies have found that content is relevant to the level of distraction $[12,15]$. Low pixel resolution of the VR content influenced the experience of at least one participant 
- Table 1 Baseline characteristics.

\begin{tabular}{|c|c|c|c|}
\hline & $\begin{array}{l}\text { Control (non-VR) } \\
(n=9)\end{array}$ & $\begin{array}{l}\text { Intervention (VR) } \\
(n=10)\end{array}$ & $P$ value \\
\hline Age (years) ${ }^{1}$ & $64[47.5 ; 67.5]$ & $65[62,67]$ & $0.414^{2}$ \\
\hline Gender (male:female) & $4: 5$ & $4: 6$ & $1.000^{3}$ \\
\hline \multicolumn{4}{|l|}{ RAND-36 } \\
\hline Physical functioning ${ }^{1}$ & $90[70,100]$ & $82.5[72.5 ; 95]$ & $0.549^{2}$ \\
\hline Role limitations due to physical health ${ }^{1}$ & $87.5[68.75 ; 100]$ & $68.75[50 ; 84.38]$ & $0.156^{2}$ \\
\hline Role limitations due to emotional problems ${ }^{1}$ & $100[53.13 ; 81.25]$ & $83.33[47.92 ; 100]$ & $0.133^{2}$ \\
\hline Energy/ fatique ${ }^{1}$ & $75[72.5 ; 90]$ & $59.38[48.44 ; 81.25]$ & $0.497^{2}$ \\
\hline Emotional well-being ${ }^{1}$ & $85[75,100]$ & $75[50 ; 81.25]$ & $0.113^{2}$ \\
\hline Social functioning ${ }^{1}$ & $100[73.47 ; 94.9]$ & $81.25[62.5 ; 100]$ & $0.113^{2}$ \\
\hline Pain $^{1}$ & $89.79[73.47 ; 67.5]$ & $72.45[67.35 ; 100]$ & $0.497^{2}$ \\
\hline General health ${ }^{1}$ & $55[35 ; 67.5]$ & $57.5[52.5 ; 66.25]$ & $0.497^{2}$ \\
\hline Health change ${ }^{1}$ & $50[25 ; 62.5]$ & $37.5[25,50]$ & $0.549^{2}$ \\
\hline Number of previous colonoscopy ${ }^{1}$ & $2.5[1.75 ; 5]$ & $2[1.25 ; 3.75]$ & $0.515^{2}$ \\
\hline Level of education & & & $0.733^{4}$ \\
\hline Primary school & $0(0)$ & $0(0)$ & \\
\hline Lower vocational education & $0(0)$ & $10(1)$ & \\
\hline Lower general secondary school & $0(0)$ & $0(0)$ & \\
\hline Intermediate general secondary school & $11.1(1)$ & $10(1)$ & \\
\hline Intermediate vocational education & $22.2(2)$ & $20(2)$ & \\
\hline Upper general secondary school & $22.2(2)$ & $10(1)$ & \\
\hline Higher vocational education & $33.3(3)$ & $50(5)$ & \\
\hline University & $11.1(1)$ & $0(0)$ & \\
\hline Prior experience with VR (yes) \% (n) & $22.2(2)$ & $30(3)$ & $1.000^{3}$ \\
\hline $\begin{array}{l}\text { VR, virtual reality } \\
{ }^{1} \text { Variables are denoted as median (interquartile r } \\
{ }^{2} \text { Mann-Whitney } U \text { test. } \\
{ }^{3} \text { Fisher's Exact test. } \\
{ }^{4} \text { Chi-square test. }\end{array}$ & & & \\
\hline
\end{tabular}

and previous studies hhave shown that low-resolution videos reduce the quality of the experience [38].

The literature on VR for patients in endoscopy is scarce. A retrospective study of 190 patients found that VR allowed unsedated transnasal gastroscopy in children and young adults. In this study, VR-assisted transnasal gastroscopy was safe and cost-effective for staging of eosinophilic esophagitis [39]. The argument has been made that VR makes it possible to avoid sedation for colonoscopy, which fuels patient experience [40]. Therefore it is probable that in selected patients, VR during colonoscopy will be the preferred option [29].

\section{Strengths and limitations}

Our study was performed in a real-life setting and a representative sample of patients, which add to its external validity. By using Samsung Gear VR to provide distraction, we chose a widely available and relatively inexpensive VR device, enhancing the generalizability of the results.

Our study also comes with limitations. First, the small sample size does not allow robust statements on clinically relevant endpoints like reducing anxiety or pain or improving patient satisfaction. Also, recent literature suggests that our sample size computation carries the risk of overestimation of the required sample size when designing a main trial to confirm our results [41]. Second, the physicians who performed the procedure were not blinded, because the patients in the control group did not wear VR glasses. Although we did not observe a differ- 
- Table 2 Procedure characteristics.

\begin{tabular}{|c|c|c|c|}
\hline & $\begin{array}{l}\text { Control (non VR) } \\
(n=9)\end{array}$ & $\begin{array}{l}\text { Intervention (VR) } \\
(n=10)\end{array}$ & $P$ value \\
\hline Dose midazolam, in mg ${ }^{1}$ & $\begin{array}{l}2.5[2.5 ; 3] \\
\min 2 ; \max 3.75\end{array}$ & $\begin{array}{l}2.5[2.38 ; 3] \\
\min 2.5 ; \max 3\end{array}$ & $0.842^{2}$ \\
\hline Dose alfentanyl, in $\mathrm{mg}^{1}$ & $\begin{array}{l}0.25[0.25 ; 0.50] \\
\min 0.25 ; \max 0.50\end{array}$ & $\begin{array}{l}0.25[0.25 ; 0.5] \\
\min 0.25 ; \max 0.50\end{array}$ & $0.278^{2}$ \\
\hline Completed colonoscopies \% ( $n$ ) & $100(9)$ & $90(9)$ & $1.000^{3}$ \\
\hline Patient acceptance of VR glasses \% ( $n$ ) & $\mathrm{n} / \mathrm{a}$ & $100(10)$ & $\mathrm{n} / \mathrm{a}$ \\
\hline Time to reach the cecum, in minutes ${ }^{1}$ & $\begin{array}{l}10.48[8.65 ; 13.80] \\
\min 6.10 ; \max 19.00\end{array}$ & $\begin{array}{l}6.83[5.75 ; 10.77] \\
\min 2.66 ; \max 11.92\end{array}$ & $0.094^{2}$ \\
\hline Time to complete procedure, in minutes ${ }^{1}$ & $\begin{array}{l}21.20[19.72 ; 35.15] \\
\min 19.18 ; \max 44.07\end{array}$ & $\begin{array}{l}22.60[16.25 ; 25.45] \\
\min 9.95 ; \max 26.43\end{array}$ & $0.340^{2}$ \\
\hline \multicolumn{4}{|c|}{$\begin{array}{l}\text { VR, virtual reality } \\
{ }^{1} \text { Variables are denoted as median (interquartile range). } \\
{ }^{2} \text { Mann-Whitney U test. } \\
{ }^{3} \text { Fisher's Exact test. }\end{array}$} \\
\hline
\end{tabular}

- Table 3 Pain, patient comfort and anxiety results.

\begin{tabular}{|c|c|c|c|}
\hline & $\begin{array}{l}\text { Control (non-VR) } \\
(n=9)\end{array}$ & $\begin{array}{l}\text { Intervention (VR) } \\
(n=10)\end{array}$ & $P$ value \\
\hline Pain score (pre-procedure) ${ }^{1}$ & $0[0,3]$ & $0[0 ; 1.75]$ & $0.968^{2}$ \\
\hline Pain score (during procedure) ${ }^{1}$ & $3[1,4]$ & $3[1.5 ; 5.5]$ & $0.661^{2}$ \\
\hline Gloucester comfort scale \% (n) & & & $0.699^{3}$ \\
\hline Comfortable & $44.4(4)$ & $40(4)$ & \\
\hline Minimal & $44.4(4)$ & $30(3)$ & \\
\hline Mild & $11.1(1)$ & $20(2)$ & \\
\hline Moderate & $0(0)$ & $0(0)$ & \\
\hline Severe & $0(0)$ & $10(1)$ & \\
\hline STATE (Pre-procedure) ${ }^{1}$ & $49[48,50]$ & $48.5[45.75 ; 50.25]$ & $0.497^{2}$ \\
\hline TRAIT $^{1}$ & $29[21 ; 36.5]$ & $35[28 ; 41.5]$ & $0.156^{2}$ \\
\hline STATE (Post-procedure) ${ }^{1}$ & $50[48 ; 52.5]$ & $50[47.75 ; 51.25]$ & $0.549^{2}$ \\
\hline \multicolumn{4}{|c|}{$\begin{array}{l}\text { VR, virtual reality } \\
{ }^{1} \text { Variables are denoted as median (interquartile range). } \\
{ }^{2} \text { Mann-Whitney U test. } \\
{ }^{3} \text { Chi-square test. }\end{array}$} \\
\hline
\end{tabular}

- Table 4 Satisfaction results.

\begin{tabular}{|c|c|c|c|}
\hline & $\begin{array}{l}\text { Control (non-VR) } \\
(n=9)\end{array}$ & $\begin{array}{l}\text { Intervention (VR) } \\
(n=10)\end{array}$ & $P$ value \\
\hline Patient satisfaction ${ }^{1}$ & $9[8,10]$ & $9[6.5 ; 10]$ & $0.905^{2}$ \\
\hline NPS 1 & $9[8,10]$ & $9[7.75 ; 10]$ & $0.905^{2}$ \\
\hline Willingness to return ${ }^{1}$ & $9[7.5 ; 10]$ & $9[6.75 ; 10]$ & $0.720^{2}$ \\
\hline $\begin{array}{l}\text { VR, virtual reality. } \\
\text { NPS, net promoter score. } \\
{ }^{1} \text { Variables are denoted as median [interquartile range]. } \\
{ }^{2} \text { Mann-Whitney U test. }\end{array}$ & & & \\
\hline
\end{tabular}


ence in administration of drugs in the control and intervention groups, this could have affected the choice and dose of sedatives. The ideal set-up is a direct comparison of sedation vs VR, instead of VR combined with sedation as done in our pilot.

We used patient-reported measures for pain and comfort after patients were recovering from sedative administration. The post-amnesia effect of midazolam might have had some effect, but the Gloucester scale rated by the nursing staff revealed no differences between groups.

Because of the low costs of the VR device, VR distraction may easily be deployed in colonoscopy. There are several technical shortcomings such as low resolution, orientation, and content, which if resolved may improve the distractive effect and help ensure enhanced patient comfort and satisfaction.

To achieve a maximal immersive effect, VR content must be developed that provides specific targeted distraction for colonoscopy, such as relaxing colors, relaxing music, and properly selected visualizations.

\section{Conclusion}

In summary, patients accepted VR distraction undergoing colonoscopy, without compromising the technical success of the procedure. Future studies are justified to evaluate the possible substitution of sedation with VR. Patients reported that the VR experience was pleasant and distracting, facilitating recruitment for these trials.

\section{Acknowledgements}

The authors would like to acknowledge Dr. Fia Voogd (FV) and Dr. Björn van Heumen (BvH) as the participating endoscopists in this trial.

\section{Competing interests}

Funding for the trial was supported by Radboudumc. Visyon (supplier of hardware) had no role in the funding of this trial, nor in design and conduct of the study or in the writing and submission of the manuscript.

\section{References}

[1] Peterse EFP, Meester RGS, Siegel RL et al. The impact of the rising colorectal cancer incidence in young adults on the optimal age to start screening: Microsimulation analysis I to inform the American Cancer Society colorectal cancer screening guideline. Cancer 2018; 124: 2964-2973

[2] Lauriola M, Tomai M, Palma R et al. Intolerance of uncertainty and anxiety-related dispositions predict pain during upper endoscopy. Frontiers Psychol 2019; 10: 1112

[3] Brandt L]. Patients' attitudes and apprehensions about endoscopy: how to calm troubled waters. Am J Gastroenterol 2001; 96: 280

[4] Bynum SA, Davis JL, Green BL et al. Unwillingness to participate in colorectal cancer screening: examining fears, attitudes, and medical mistrust in an ethnically diverse sample of adults 50 years and older. Am J Health Promotion 2012; 26: 295-300
[5] Shafer LA, Walker JR, Waldman C et al. Factors associated with anxiety about colonoscopy: the preparation, the procedure, and the anticipated findings. Digest Dis Sci 2018; 63: 610-618

[6] Cohen LB, Delegge MH, Aisenberg J et al. AGA Institute review of endoscopic sedation. Gastroenterology 2007; 133: 675-701

[7] Cote GA, Hovis RM, Ansstas MA et al. Incidence of sedation-related complications with propofol use during advanced endoscopic procedures. Clin Gastroenterol Hepatol 2010; 8: 137-142

[8] Lee LA, Caplan RA, Stephens LS et al. Postoperative opioid-induced respiratory depression: a closed claims analysis. Anesthesiology 2015; 122: 659-665

[9] Kollmann CM, Schmiegel W, Brechmann T. Gastrointestinal endoscopy under sedation is associated with pneumonia in older inpatientsresults of a retrospective case-control study. United European Gastroenterol J 2018; 6: 382-390

[10] Wernli KJ, Brenner AT, Rutter CM et al. Risks associated with anesthesia services during colonoscopy. Gastroenterology 2016; 150: 888894; quiz e818

[11] Lin OS. Sedation for routine gastrointestinal endoscopic procedures: a review on efficacy, safety, efficiency, cost and satisfaction. Intest Res 2017; 15: 456-466

[12] El Hassan H, McKeown K, Muller A. Clinical trial: music reduces anxiety levels in patients attending for endoscopy. Aliment Pharmacol Therap 2009; 30: 718-724

[13] Lee D, Chan A, Wong $S$ et al. Can visual distraction decrease the dose of patient-controlled sedation required during colonoscopy? A prospective randomized controlled trial Endoscopy 2004; 36: 197-201

[14] Martindale F, Mikocka-Walus AA, Walus BP et al. The effects of a designer music intervention on patients' anxiety, pain, and experience of colonoscopy: a short report on a pilot study. Gastroenterol Nursing 2014; 37: 338-342

[15] Umezawa S, Higurashi T, Uchiyama S et al. Visual distraction alone for the improvement of colonoscopy-related pain and satisfaction. World J Gastroenterol 2015; 21: 4707

[16] Nomura T, Higuchi K, Yu H et al. Slow-wave photic stimulation relieves patient discomfort during esophagogastroduodenoscopy. J Gastroenterol Hepatol 2006; 21: 54-58

[17] Fanti L, Gemma M, Passaretti S et al. Electroacupuncture analgesia for colonoscopy: a prospective, randomized, placebo-controlled study. Am J Gastroenterol 2003; 98: 312-316

[18] Lembo T, Fitzgerald L, Matin K et al. Audio and visual stimulation reduces patient discomfort during screening flexible sigmoidoscopy. Am J Gastroenterol 1998; 93: 1113-1116

[19] Sjolander A, Jakobsson Ung E, Theorell T et al. Hospital design with nature films reduces stress-related variables in patients undergoing colonoscopy. HERD 2019; 12: 186-196

[20] Gold JI, Belmont KA, Thomas DA. The neurobiology of virtual reality pain attenuation. CyberPsychol Behav 2007; 10: 536-544

[21] Hoffman HG, Richards TL, Van Oostrom T et al. The analgesic effects of opioids and immersive virtual reality distraction: evidence from subjective and functional brain imaging assessments. Anes Analg 2007; 105: 1776-1783

[22] Chapman CR, Nakamura Y. Hypnotic analgesia: A constructivist framework. Int J Clin Exp Hypn 1998; 46: 6-27

[23] McCaul KD, Malott JM. Distraction and coping with pain. Psychol Bull 1984; 95: 516

[24] Dascal J, Reid M, IsHak WW et al. Virtual reality and medical inpatients: a systematic review of randomized, controlled trials. Innov Clin Neurosci 2017; 14: 14-21

[25] Jeffs D, Dorman D, Brown S et al. Effect of virtual reality on adolescent pain during burn wound care. J Burn Care Res 2014; 35: 395-408 
[26] Maani CV, Hoffman HG, Morrow M et al. Virtual reality pain control during burn wound debridement of combat-related burn injuries using robot-like arm mounted VR goggles. J Trauma 2011; 71: S125

[27] Das DA, Grimmer KA, Sparnon AL et al. The efficacy of playing a virtual reality game in modulating pain for children with acute burn injuries: a randomized controlled trial. BMC Ped 2005; 5: 1

[28] Furman E, Jasinevicius TR, Bissada NF et al. Virtual reality distraction for pain control during periodontal scaling and root planing procedures. J Am Dental Assoc 2009; 140: 1508-1516

[29] Blokzijl S], Lamberts KF, van der Waaij LA et al. Short article: Willingness to undergo colonoscopy with virtual reality instead of procedural sedation and analgesia. Europ J Gastroenterol Hepatol 2019; 31: 334339

[30] Julious SA. Sample size of 12 per group rule of thumb for a pilot study. Pharm Stat 2005; 4: 287-291

[31] Vander Zee KI, Sanderman R, Heyink JW et al. Psychometric qualities of the RAND 36-Item Health Survey 1.0: a multidimensional measure of general health status. Int J Behav Med 1996; 3: 104-122

[32] Breivik $\mathrm{H}$, Borchgrevink P, Allen S et al. Assessment of pain. Br J Anaesthesia 2008; 101: 17-24

[33] Spielberger CD. Manual for the State-Trait anxiety inventory (form Y). Plato Alto, CA: Consulting Psychologists Press; 1987

[34] Rostom A, Ross ED, Dubé $C$ et al. Development and validation of a nurse-assessed patient comfort score for colonoscopy. Gastrointest Endosc 2013; 77: 255-261
[35] Reichheld FF. The one number you need to grow. Harvard Bus Rev 2003; 81: 46-55

[36] Kipping B, Rodger S, Miller K et al. Virtual reality for acute pain reduction in adolescents undergoing burn wound care: a prospective randomized controlled trial. Burns 2012; 38: 650-657

[37] Morris LD, Louw QA, Crous LC. Feasibility and potential effect of a lowcost virtual reality system on reducing pain and anxiety in adult burn injury patients during physiotherapy in a developing country. Burns 2010; 36: 659-664

[38] McCarthy JD, Sasse MA, Miras D. Sharp or smooth? comparing the effects of quantization vs. frame rate for streamed video Vienna, Austria: Proceedings of the SIGCHI Conference on Human Factors in Computing Systems; 2004

[39] Nguyen N, Lavery WJ, Capocelli KE et al. Transnasal endoscopy in unsedated children with eosinophilic esophagitis using virtual reality video goggles. Clin Gastroenterol Hepatol 2019: doi:10.1016/j. cgh.2019.01.023

[40] Terruzzi V, Paggi S, Amato A et al. Unsedated colonoscopy: A neverending story. World J Gastrointest Endosc 2012; 4: 137-141

[41] Whitehead AL, Julious SA, Cooper CL et al. Estimating the sample size for a pilot randomised trial to minimise the overall trial sample size for the external pilot and main trial for a continuous outcome variable. Stat Methods Med Res 2016; 25: 1057-1073 